\title{
PCSK9 regulates apoptosis in human lung adenocarcinoma A549 cells via endoplasmic reticulum stress and mitochondrial signaling pathways
}

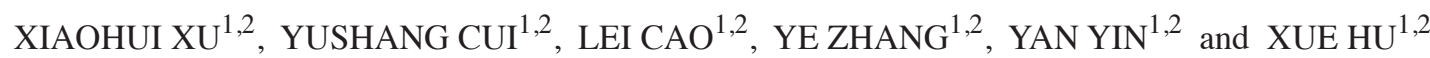 \\ ${ }^{1}$ Department of Thoracic Surgery, Peking Union Medical College Hospital; ${ }^{2}$ Graduate School of \\ Peking Union Medical College, Chinese Academy of Medical Sciences, Beijing 100730, P.R. China
}

Received December 5, 2015; Accepted December 9, 2016

DOI: $10.3892 / \mathrm{etm} .2017 .4218$

\begin{abstract}
Proprotein convertase subtilisin/kexin type 9 (PCSK9) is a member of the subtilisin family of PCs that encodes a neural apoptosis-regulated convertase 1. However, the precise role of PCSK9 in lung cancer cell apoptosis has remained elusive. In the present study, A549 human lung adenocarcinoma cells were transfected with PCSK9 small interfering (si)RNA to investigate the underlying mechanisms of apoptosis. The results indicated that PCSK9 siRNA exhibited anti-tumor activity by inducing apoptosis as determined by a Cell Counting Kit- 8 and Hoechst staining analysis. In addition, PCSK9 siRNA significantly increased apoptosis of A549 cells in part via activation of caspase-3 and downregulation of the anti-apoptotic proteins survivin and X-linked inhibitor of apoptosis protein. Moreover, the results demonstrated that perturbations in the mitochondrial membrane were associated with the deregulation of the $\mathrm{Bax} / \mathrm{Bcl}-2$ ratio, which led to the release of cytochrome $c$ after PCSK9 siRNA transfection. In addition, PCSK9 siRNA also induced endoplasmic reticulum stress (ERS) by increasing the levels of $78 \mathrm{kDa}$ glucose-regulated protein (GRP78), GRP94, phosphorylated protein kinase R-like ER kinase and phosphorylated eukaryotic initiation factor $2 \alpha$. Therefore, these results demonstrated that PCSK9 siRNA may exert its anti-tumor activity through inducing mitochondrial dysfunction and ERS-associated cell death in A549 cells.
\end{abstract}

\section{Introduction}

Lung cancer is a disease that severely threatens human health and its incidence shows yearly increases (1). Lung

Correspondence to: Dr Yushang Cui, Department of Thoracic Surgery, Peking Union Medical College Hospital, 1 Shuaifuyuan, Dongcheng, Beijing 100730, P.R. China

E-mail: cuiyshang@yeah.net

Key words: PCSK9, lung adenocarcinoma, apoptosis, endoplasmic reticulum stress, mitochondrial dysfunction cancer accounts for the highest number of cancer-associated mortalities in women and men worldwide (2). Metastasis and recurrence are the primary cause of death in patients with lung cancer, and almost $90 \%$ of lung cancer patients succumb to tumor metastasis (3). Unrestricted proliferation of cancer cells leads to tumor enlargement and subsequent compression on peripheral organs. Inhibition of tumor cells is an effective measure of treating malignant tumors. Although chemotherapy is an important component of the first-line therapies for lung adenocarcinoma (LAD), chemoresistance represents a predominant obstacle towards chemotherapeutic treatment of LAD. Induction of apoptosis has been found to be an effective means of treating cancer, including non-small-cell lung cancer (4). Therefore, in-depth study of the molecular mechanisms of lung cancer cell proliferation and apoptosis is necessary.

Proprotein convertase subtilisin/kexin type 9 (PCSK9) belongs to the family of PCs and encodes a neural apoptosis-regulated convertase 1 (5).PCSK9 was recently discovered to have two major biological functions: Maintenance of plasma lipid homeostasis by regulation of low-density lipoprotein receptors and regulation of neuronal apoptosis (6-8). Gain- and loss-of-function mutations in the PCSK9 gene are associated with hyper- and hypocholesterolemia, respectively (9-11). Therefore, PCSK9 inhibition is used as a promising therapy to treat hypercholesterolemia (12). PCSK9 is also involved in numerous biological processes. Microarray studies showed that overexpression of PCSK9 leads to the dysregulation of numerous pathways, including those regulating the cell cycle, apoptosis and inflammation $(13,14)$. In vivo studies also suggested that PCSK9 is implicated in these processes $(15,16)$. Sun et al (17) demonstrated that PCSK9 deficiency reduced liver metastasis by its ability to lower cholesterol levels and possibly by enhancing tumor necrosis factor $\alpha$-mediated apoptosis.

As biological processes such as cell cycle and proliferation are modified in cancer, the present study hypothesized that PCSK9 may regulate cancer cell apoptosis. To the best of our knowledge, no studies regarding the possible role of PCSK9 in the development of lung cancer are available. The aim of the present study was to identify the function of PCSK9 during apoptosis of lung cancer cells. It was found that PCSK9 small 
interfering (si)RNA significantly increased the apoptosis of A549 cells. The results suggested that inhibition of PCSK9 induces apoptosis and inhibits proliferation of LAD cells.

\section{Materials and methods}

Reagents and antibodies. Rabbit anti-human cleaved (c)-caspase-3 (cat. no. 25546-1-AP, 1:2,000), B-cell lymphoma 2 (Bcl-2; cat. no. 12789-1-AP; 1:2,000), Bcl-2-associated X protein (Bax; cat. no. 23931-1-AP; 1:2,000), $78 \mathrm{kDa}$ glucose-regulated protein (GRP78; cat. no. 11587-1-AP; 1:2,000), GRP94 (cat. no. 14700-1-AP; 1:2,000), protein kinase R-like ER kinase (PERK; cat. no. 24390-1-AP, 1:2,000), cytochrome $c$ oxidase (COX) IV (cat. no. 11242-1-AP; 1:2,000), X-linked inhibitor of apoptosis protein (XIAP; cat. no. 10037, 1:2,000) and survivin (cat. no. 10508-1-AP; 1:2,000) antibodies, as well as mouse anti-GAPDH (cat. no. 60004; 1:2,000) antibody were purchased from Proteintech (Wuhan, China). Rabbit anti-human PCSK9 (cat. no. ab181142; 1:3,000) and cytochrome $c$ (cat. no. ab133504; 1:3,000) antibodies were purchased from Abcam (Cambridge, UK). Rabbit anti-human phosphorylated (p)-PERK (cat. no. \#5683; 1:3,000), eukaryotic initiation factor (eIF) $2 \alpha$ (cat. no. \#5169; 1:3,000) and p-eIF2 $\alpha$ (cat. no. 3398; 1:3,000) antibodies were purchased from Cell Signaling Technology, Inc. (Danvers, MA, USA). Horseradish peroxidase (HRP)-conjugated goat anti-rabbit immunoglobulin G (cat. no. 10285-1-AP; 1:2,000) or mouse immunoglobulin G (cat. no. 16402; 1:2,000) were purchased from Proteintech. Cell Counting Kit (CCK)-8 and Hoechst 33258 were purchased from Beyotime Institute of Biotechnology (Haimen, China). Other reagents were of analytical grade.

Cell culture and RNA interference. The A549 human LAD cell line was obtained from the American Type Culture Collection (Manassas, VA,USA).Cells were routinely grown in RPMI-1640 medium (Hyclone; GE Healthcare, Chalfont, UK) containing $10 \%$ fetal bovine serum (FBS; Hyclone), $100 \mathrm{U} / \mathrm{ml}$ penicillin (Sigma-Aldrich, Merck-Millipore, Darmstadt, Germany) and $100 \mu \mathrm{g} / \mathrm{ml}$ streptomycin (Sigma-Aldrich) at $37^{\circ} \mathrm{C}$ in a humidified atmosphere containing $5 \% \mathrm{CO}_{2}$. The medium was replaced every 2-3 days and upon reaching $80 \%$ confluence, they were passaged at a 1:2 ratio.

A549 cells were transfected with $100 \mathrm{nM}$ PCSK9 siRNA (cat. no. sc-45482; Santa Cruz Biotechnology, Inc., Dallas, TX, USA; Genbank ID for PCSK9: NM_174936) or control siRNA (scrambled siRNA, a universal negative control; cat. no. sc-37007; Santa Cruz Biotechnology, Inc., Dallas, TX, USA) with GeneSilencer siRNA transfection reagent (Genlantis, San Diego, CA, USA), according to the manufacturer's instructions. At $48 \mathrm{~h}$ after transfection, the efficiency of siRNA-mediated PCSK9 knockdown was determined by western blot analysis.

Cell proliferation assay. A549 cells were seeded into each well of a 96-well plate at a density of $5 \times 10^{3}$ cells/well in culture medium. After $24 \mathrm{~h}$ incubation, cells were transfected with PCSK9 siRNA or control siRNA for 12, 24, 36 and $48 \mathrm{~h}$ as described above, followed by the addition of $10 \mu \mathrm{l}$ CCK-8 solution. The cells were then incubated for $3 \mathrm{~h}$ at $37^{\circ} \mathrm{C}$. Absorbance was measured at 450 and $650 \mathrm{~nm}$ using a spectrophotometer (Nanodrop 2000 UV-VIS; Thermo Fisher Scientific, Inc., Wilmington, DE, USA). The experiments were performed in triplicate.

Morphological analysis following Hoechst 33258 staining. A549 cells were seeded in 24 -well plates $\left(6 \times 10^{4}\right.$ cells/well) overnight and transfected with PCSK9 siRNA or control siRNA for $48 \mathrm{~h}$. The cells were then fixed and stained with Hoechst 33258 in the dark at $4^{\circ} \mathrm{C}$ overnight. Apoptotic cells were visualized using a fluorescence microscope (DM 6000; Leica microsystems GmbH, Wetzler, Germany).

Preparation of mitochondria and cytosol. A mitochondria/cytosol kit (Beyotime Institute of Biotechnology) was used to isolate mitochondria and cytosol according to the manufacturer's instructions. Following the aforementioned protocol of transfection, $5 \times 10^{7}$ cells were collected by centrifugation at $600 \mathrm{xg}$ for $5 \mathrm{~min}$ at $4^{\circ} \mathrm{C}$, washed twice with ice-cold PBS and then resuspended in $500 \mu l$ isolation buffer containing protease inhibitors for $10 \mathrm{~min}$ on ice. The cells were mechanically homogenized with a Dunce grinder. The unbroken cells, debris and nuclei were discarded by centrifugation at $800 \times g$ for $10 \mathrm{~min}$ at $4^{\circ} \mathrm{C}$. The supernatants were centrifuged at $12,000 \times \mathrm{g}$ for $15 \mathrm{~min}$ at $4^{\circ} \mathrm{C}$. The supernatant containing the cytosol was collected and the pellet fraction containing the mitochondria was dissolved in $50 \mu 1$ lysis buffer.

Western blot analysis. A549 cells were transfected as described above. Cells were harvested and lysed for $10 \mathrm{~min}$ in ice-cold lysis buffer [50 mM Tris- $\mathrm{HCl} \mathrm{pH} 8.0,140 \mathrm{mM} \mathrm{NaCl}$, $1.5 \mathrm{mM} \mathrm{MgCl}{ }_{2}$ and $0.5 \%$ Nonidet P-40 with complete protease inhibitor cocktail (Roche, Mannheim, Germany)]. The lysates were further centrifuged at $12,000 \times \mathrm{g}$ for $15 \mathrm{~min}$ at $4^{\circ} \mathrm{C}$ and the supernatants were then collected in new tubes. The protein concentration was measured using a bicinchoninic acid protein assay kit (Dingguo, Beijing, China). Protein samples were boiled for $5 \mathrm{~min}$ in the presence of $5 \mathrm{X}$ SDS-PAGE loading buffer. Equal amounts of proteins were subjected to $12 \%$ SDS-PAGE and then electrotransferred onto PVDF membranes (Millipore, Billerica, MA, USA). The membranes were blocked for $1 \mathrm{~h}$ in Tris-buffered saline $(25 \mathrm{mM}$ Tris at $\mathrm{pH} 7.5,150 \mathrm{mM} \mathrm{NaCl}$ and $0.05 \%$ Tween-20) containing $5 \%$ nonfat milk powder, and incubated overnight at $4^{\circ} \mathrm{C}$ with the indicated antibodies. After washing, blots were incubated for $1 \mathrm{~h}$ at $37^{\circ} \mathrm{C}$ with HRP-conjugated anti-rabbit or anti-mouse secondary antibodies. Following further washing, the blots were revealed using the ECL Plus detection system (Thermo Fisher Scientific, Inc.) under conditions recommended by the manufacturer. Images were captured directly by the Gel 3100 chemiluminescent and fluorescent imaging system (Sage Creation Science Co., Ltd., Beijing, China). Quantification of band densities was performed using Quantity One software version 2.0 (Bio-Rad Laboratories, Inc., Hercules, CA, USA) with normalization to the GAPDH signal. The level of proteins of interest in the siRNA group was expressed relative to that in the control siRNA group.

Statistical analysis. Values are expressed as the mean \pm standard deviation. Differences were analyzed using Student's 
A

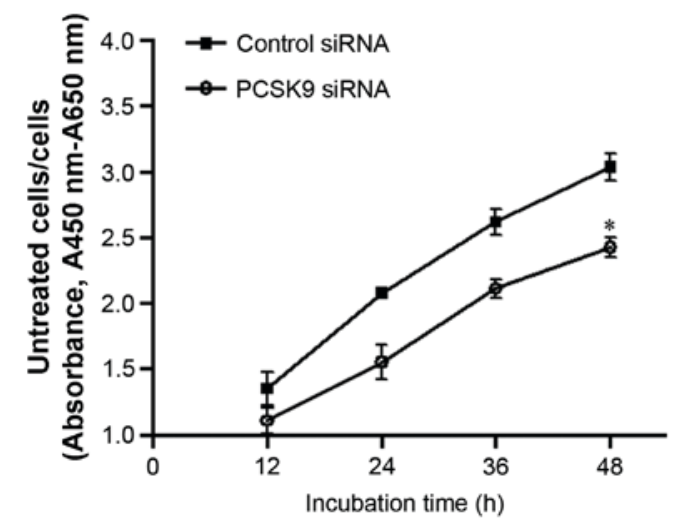

B

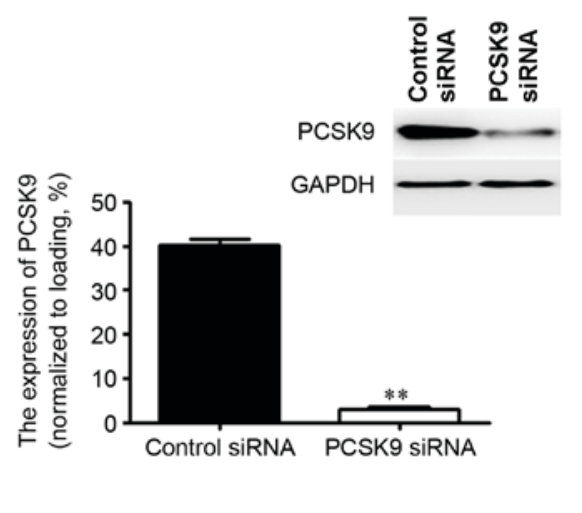

Figure 1. Proliferation of A549 cells was inhibited by PCSK9 siRNA. (A) A549 cells were transfected with PCSK9 siRNA or control siRNA for the indicated durations. Cell proliferation was examined using the Cell Counting Kit-8 assay. The optical density of each well was measured at 450 and $650 \mathrm{~nm}$ using a microplate reader. Values are expressed as the mean \pm standard deviation of A450-A650 at 12, 24, 36 and $48 \mathrm{~h}$ of siRNA transfection (each concentration was tested in triplicate). (B) Expression of PCSK9 in A549 cells following siRNA transfection. The level of PCSK9 was significantly decreased following RNA interference. GAPDH was included as a loading control. Values are expressed as the mean \pm standard deviation $(n=3)$. ${ }^{*} \mathrm{P}<0.05$ vs. control siRNA; ${ }^{* *} \mathrm{P}<0.001$ vs. control siRNA group. A650 was assigned as internal control; A450 was assigned as the examining wavelength. siRNA, small interfering RNA; A650, absorbance at $650 \mathrm{~nm}$; A450, absorbance at $450 \mathrm{~nm}$; PCSK9, proprotein convertase subtilisin/kexin type 9.

A

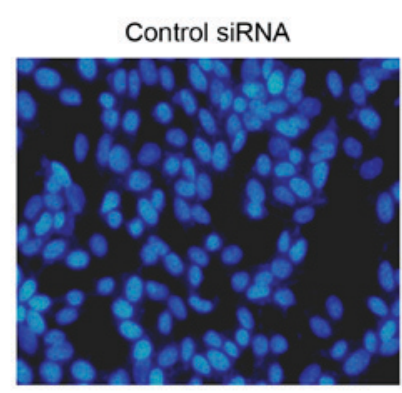

B
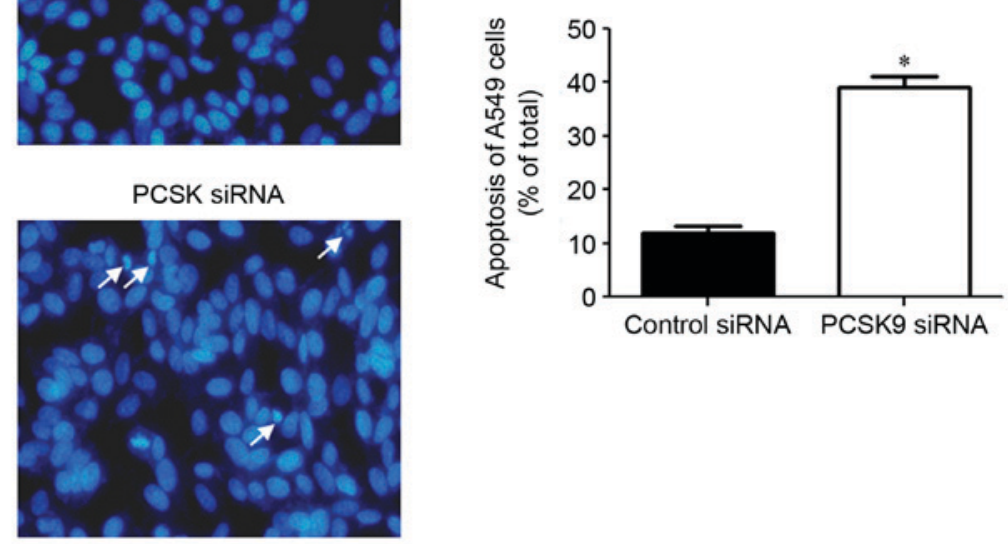

Figure 2. PCSK9 siRNA significantly increased apoptosis of A549 cells. (A) After $48 \mathrm{~h}$ transfection, cells were incubated with Hoechst 33258 staining buffer. Healthy cells showed round and intact nuclei, whereas apoptotic cells exhibited nuclear karyopyknosis or fragmentation as indicated by arrows (magnification, x200). (B) Quantification of apoptotic cells by counting the number of apoptotic cells three times. At least six fields and six samples were examined for each group. The cells transfected with PCSK9 siRNA exhibited higher ratio of apoptotic cells as compared to the control siRNA group. Values are expressed as the mean \pm standard deviation $(n=3) .{ }^{*} \mathrm{P}<0.05$ vs. control siRNA group. PCSK9, proprotein convertase subtilisin/kexin type 9; siRNA, small interfering RNA.

t-test and one-way analysis of variance to determine the level of significance. $\mathrm{P}<0.05$ or 0.001 was considered to indicate a statistically significant difference. Statistical analysis was performed using SPSS 19.0 software (IBM SPSS, Armonk, NY, USA).

\section{Results}

Effect of PCSK 9 on A549 cell proliferation. A549 cell proliferation was examined using a CCK- 8 assay performed subsequent to transfection with PCSK9 siRNA or control siRNA. As shown in Fig. 1A, PCSK9 siRNA attenuated the increase in cell proliferation that occurred in the control siRNA-treated group. Furthermore, the cell proliferation at $48 \mathrm{~h}$ after transfection with PCSK9 siRNA was significantly reduced compared with that in the control siRNA group $(\mathrm{P}<0.05$; Fig. 1A). Additionally, results from western blotting indicated that PCSK9 expression was significantly decreased compared with the control siRNA group $(\mathrm{P}<0.001 ;$ Fig. $1 \mathrm{~B})$. These data imply that PCSK9 siRNA inhibits the proliferation of A549 cells. 
A

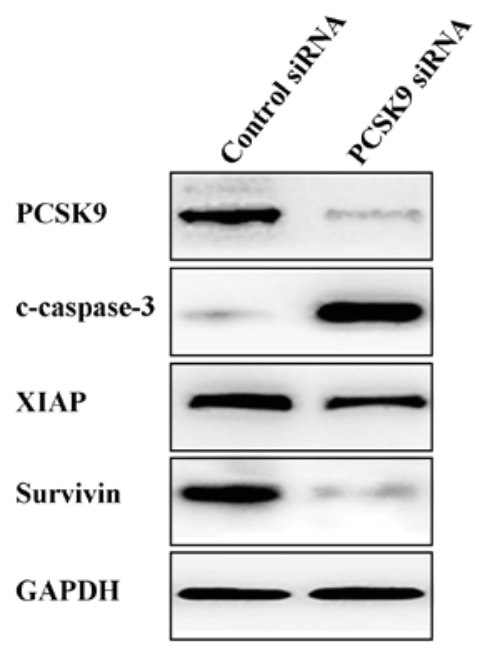

B
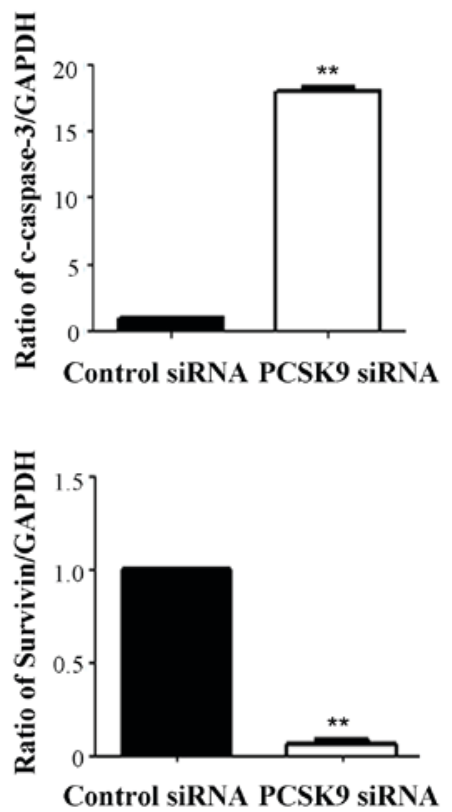

Figure 3. Western blot analysis of apoptosis-associated proteins following PCSK9 siRNA transfection. A549 cells were transfected with PCSK9 siRNA or control siRNA for $48 \mathrm{~h}$ prior to being subjected to protein extraction and western blot analysis with the indicated antibodies. (A) Representative western blot images and (B) quantified protein levels obtained by densitometric analysis. Protein levels relative to GAPDH (loading control) are presented. Levels of control siRNA were set at 1 . Values are expressed as the mean \pm standard deviation $(n=3) .{ }^{*} \mathrm{P}<0.05,{ }^{* *} \mathrm{P}<0.001$ vs. control siRNA. siRNA, small interfering RNA; PCSK9, proprotein convertase subtilisin/kexin type 9; c-caspase-3, cleaved caspase-3; XIAP, X-linked inhibitor of apoptosis protein.

PCSK9 siRNA induces apoptosis in A549 cells. In order to evaluate whether the inhibition of A549 cell proliferation by PCSK9 siRNA was associated with apoptosis, characteristic morphological changes were observed by Hoechst 33258 staining (Fig. 2). A549 cells were transfected with PCSK9 siRNA for $48 \mathrm{~h}$ and morphological changes associated with apoptosis compared with the control group were observed. In the control siRNA group, nuclei of A549 cells were round and homogeneously stained (Fig. 2A). However, PCSK9 siRNA-transfected cells exhibited evident characteristics of apoptosis, including membrane integrity loss or deformation, cell shrinkage, nuclear fragmentation and chromatin compaction of late apoptotic appearance (Fig. 2A). Counting of cells with apoptotic features revealed that the percentage of apoptotic cells was significantly increased in the PCSK9 siRNA-transfected group, as compared to that in the group transfected with negative control siRNA ( $\mathrm{P}<0.05$; Fig. $2 \mathrm{~B})$. Together, these results indicated that PCSK 9 siRNA induces apoptosis in A549 cells.

PCSK9 siRNA induces apoptosis via the caspase-dependent mitochondrial apoptotic pathway. To further assess the role of PCSK9 in A549 cell apoptosis, the expression of apoptosis-associated proteins was assessed. These included pro-apoptotic c-caspase-3, anti-apoptotic XIAP and survivin. Western blot analysis revealed that PCSK9 siRNA increased the levels of c-caspase- 3 by $\sim 17$-fold $(\mathrm{P}<0.001)$. Conversely, the levels of XIAP and survivin were decreased by $\sim 2.5$-fold $(\mathrm{P}<0.05)$ and 16-fold $(\mathrm{P}<0.001)$, respectively, following PCSK9 siRNA transfection (Fig. 3).

In order to better understand the molecular mechanisms by which PCSK9 siRNA exerts its pro-apoptotic effects, the protein expression of mediators of the mitochondrial signaling pathway was assessed. It was determined whether PCSK9 siRNA stimulates the release of cytochrome $c$ into the cytosolic fraction in A549 cells. As expected, cytochrome $c$ was re-distributed following PCSK9 siRNA transfection. The level of cytochrome $c$ in mitochondria was significantly decreased by $50 \%(\mathrm{P}<0.05$, Fig. 4A). Correspondingly, the levels of cytochrome $c$ in cytosol were increased by $190 \%$ $(\mathrm{P}<0.05$, Fig. 4B).

Since the Bcl-2 family proteins have a critical role in regulating the release of cytochrome $c$, the present study then investigated the possible involvement of Bax and Bcl-2 in the process of PCSK9 siRNA-mediated A549 cell apoptosis. As shown in Fig. 4C, the level of Bax was significantly increased and Bcl-2 was markedly decreased in PCSK9 siRNA-transfected cells. Statistical analysis showed that PCSK9 siRNA increased the ratio of $\mathrm{Bax} / \mathrm{Bcl}-2$ by $\sim 17.5$-fold $(\mathrm{P}<0.05)$.

PCSK9 siRNA induces the activation of the ER stress pathway. Since little is known regarding the effect of PCSK9 on ER stress in cultured LAD cells, the present study determined whether PCSK9 siRNA transfection induces ER stress. The protein levels of p-PERK and p-eIF2 $\alpha$, which are considered characteristic markers of ER stress, were assessed (18). As shown in Fig. 5, compared with those in the control siRNA group, the levels of p-PERK and p-eIF2 $\alpha$ were significantly increased in PCSK9 siRNA-transfected cells, while total PERK and eIF2 $\alpha$ were unchanged. The expression of GRP78 and GRP94, which serve as gatekeepers for the activation of ER stress transducers, was then assessed (18). It was determined that the expression of GRP78 and GRP94 was significantly increased by PCSK9 siRNA transfection $(\mathrm{P}<0.05$; Fig. 5). These results demonstrated that ER stress is at least partially involved in PCSK9 siRNA-induced apoptosis. 
A
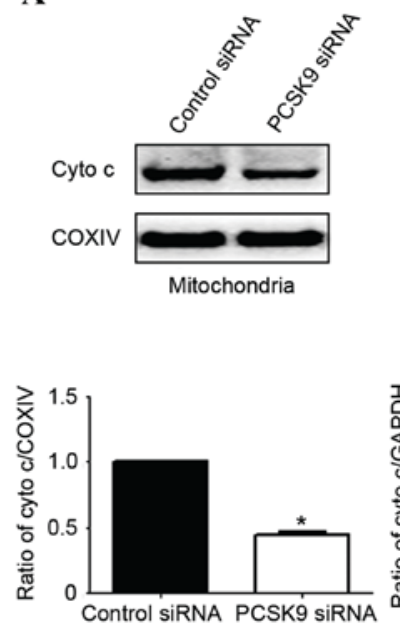

B
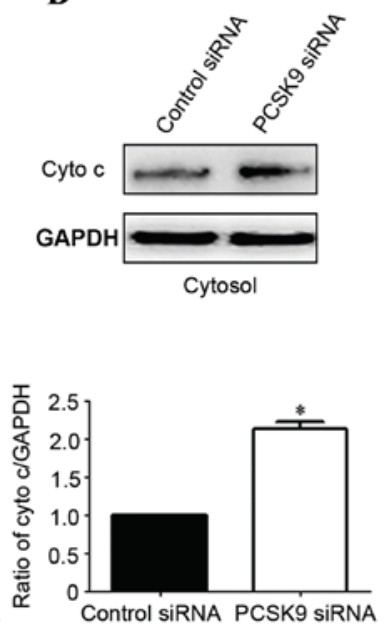

C
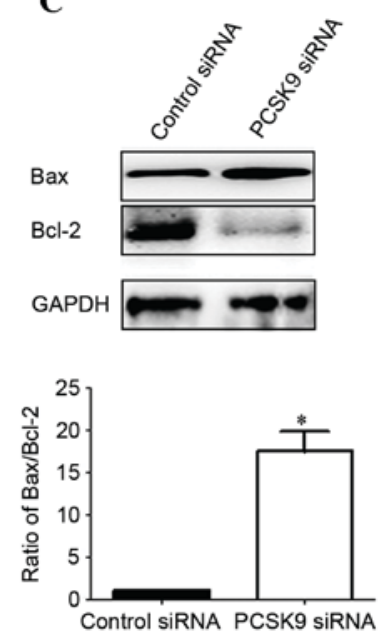

Figure 4. Regulation of mediators of the mitochondrial pathway in apoptotic A549 cells by PCSK9 siRNA. (A and B) A549 cells were transfected with PCSK9 siRNA or control siRNA for $48 \mathrm{~h}$ and the levels of cyto $c$ (A) in mitochondria (COXIV as loading control) and (B) cytosol (GAPDH as loading control) were measured by western blot analysis. (C) PCSK9 siRNA inhibited Bcl-2 expression and increased Bax expression as detected by western blot analysis. Representative western blot images are shown and bar graphs present quantified expression levels determined by densitometric analysis. Values are expressed as the mean \pm standard deviation $(\mathrm{n}=3)$. The ratio of cyto $c / \mathrm{COXIV}$ (GAPDH) in control siRNA group was assigned as ' 1 ' and the ratio of cyto $c / \mathrm{COXIV}$ (GAPDH) in PCSK9 siRNA group was assigned as proportion of control siRNA group compared with PCSK9 siRNA group. The GAPDH in C image was used to control the accounts for the samples when examining the Bax or Bcl-2 levels. "P<0.05 vs. control siRNA group. siRNA, small interfering RNA; PCSK9, proprotein convertase subtilisin/kexin type 9; Bcl-2, B-cell lymphoma 2; Bax, Bcl-2-associated X protein; cyto $c$, cytochrome $c$; COX, cyto $c$ oxidase.
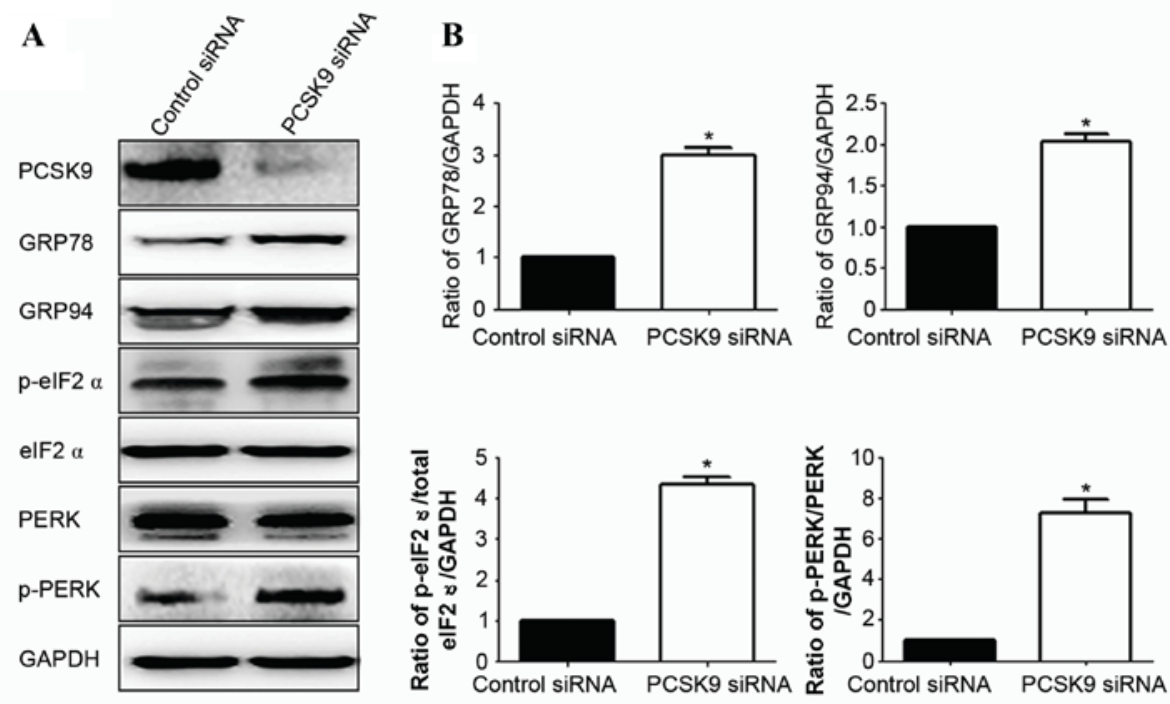

Figure 5. Effects of PCSK9 siRNA on ER stress-associated proteins in A549 cells. (A) A549 cells were transfected with PCSK9 siRNA or control siRNA for $48 \mathrm{~h}$ prior to being subjected to protein extraction and western blot analysis with the indicated antibodies. (B) Quantified protein levels relative to GAPDH (loading control) are presented. Values are expressed as the mean \pm standard deviation $(\mathrm{n}=3)$. The ratio of protein/COXIV (GAPDH) in control siRNA group was assigned as ' 1 ' and the ratio of protein/COXIV (GAPDH) in PCSK9 siRNA group was assigned as proportion of control siRNA group compared to PCSK9 siRNA group. "P<0.05 vs. control siRNA. ER, endoplasmic reticulum; siRNA, small interfering RNA; PCSK9, proprotein convertase subtilisin/kexin type 9; GRP78, 78 kDa glucose-regulated protein; p-eIF2, phosphorylated eukaryotic initiation factor 2; PERK, protein kinase R-like ER kinase.

\section{Discussion}

The PCSK9 gene is a member of the PC family and encodes the PCSK9 protein, also known as neural apoptosis-regulated convertase 1 (NARC1), which is involved in regulating apoptosis $(5,19)$. A microarray study showed that overexpression of PCSK9 ${ }^{\text {D347Y }}$ downregulated certain pro-apoptotic genes in HepG2 cells (14). In addition, the lack of PCSK9 enhanced apoptosis during liver regeneration (15). However, the exact role of PCSK9 during LAD cell apoptosis has remained elusive. The results of the present study indicated that PCSK9 siRNA inhibits the proliferation of A549 cells. Based on the results of Hoechst 33258 staining and western blot analysis, it was concluded that PCSK9 siRNA induces apoptosis in A549 cells. Thus, the present study provided the first evidence that PCSK9 has an anti-apoptotic effect in lung cancer cells. 
At present, induction of apoptosis is pursued as a strategy for killing cancer cells. Members of the IAP family, survivin and XIAP, contribute to apoptosis resistance of cancer cells (20). Thus, the present study investigated whether these apoptosis-associated proteins were involved in PCSK9 siRNA-induced apoptosis. The results confirmed the regulatory role of PCSK9 in the apoptosis of A549 cells based on the following lines of evidence: PCSK9 siRNA increased the apoptosis of A549 cells by regulating the apoptosis-associated factors c-caspase-3, XIAP and survivin. The inactivation of XIAP and survivin by PCSK9 siRNA may prevent the development and progression of cancers.

It has been well documented that Bcl-2 family proteins regulate apoptosis through a variety of pathways, primarily the mitochondrial ones (21). Bcl-2 and its homologs prevent mitochondrial membrane disruption and cytochrome $\mathrm{c}$ release, while Bax promotes these events. The Bax/Bcl-2 ratio is regarded as a determinant of the apoptotic status (22). The results of the present study demonstrated that in mitochondria, the level of cytochrome $c$ was significantly decreased, while it was increased in the cytosol, indicating the release of cytochrome $c$ from the mitochondria into the cytoplasm through mitochondrial membrane permeability increases or rupture. Furthermore, PCSK9 siRNA increased the level of Bax and decreased the level of Bcl-2, leading to an increase in the $\mathrm{Bax} / \mathrm{Bcl}-2$ ratio. These results indicated that PCSK 9 siRNA affects mitochondrial membrane stability. Taken together, these results demonstrated that PCSK9 siRNA may exert its anti-tumor activity through the mitochondrial apoptotic signaling pathway (intrinsic apoptotic pathway) in LAD cells.

Another pathway mediating apoptosis is the ER stress pathway, which may be triggered by a variety of toxic insults and ultimately leads to apoptosis (23). ER stress has been reported to induce apoptosis in various cell types via the upregulation of protein translation mediated through the PERK-eIF2 $\alpha$ pathway (24-27). For instance, upon ER stress, the ER chaperone GRP78 dissociates from the PERK and initiates transphosphorylation with subsequent activation of the kinase (28). The activated PERK then leads to phosphorylation of eIF $2 \alpha$ and subsequent expression of activating transcription factor 4 protein, which is essential for ER stress-induced apoptosis (29). The western blot results of the present study showed that PCSK9 siRNA increased the levels of GRP94, GRP78, p-PERK and p-eIF2 $\alpha$, which are vital features of the unfolded protein response and indicate that PCSK9 siRNA induced apoptosis through the ER stress signaling pathway.

In conclusion, the present study supported a role of PCSK9 in regulating apoptosis A549 cells. Further study is warranted to define the exact mechanisms by which PCSK9 regulates the apoptosis and proliferation of A549 cells. Even though the present study focused on lung cancer, a PCSK9 inhibitor, initially developed to treat hypercholesterolemia (12), may be useful in therapies directed against various types of cancer and possibly metastasis.

\section{References}

1. Liang R, Chen TX, Wang ZQ, Jin KW, Zhang LY, Yan QN Zhang HH and Wang WP: A respective analysis of the clinicopathological characteristics of large cell carcinoma of lung. Exp Ther Med 9: 197-202, 2015.
2. Siegel R, Naishadham D and Jemal A: Cancer statistics, 2013. CA Cancer J Clin 63: 11-30, 2013.

3. Mrazek AA and Chao C: Surviving cutaneous melanoma: A clinical review of follow-up practices, surveillance, and management of recurrence. Surg Clin North Am 94: 989-1002, vii-viii, 2014.

4. Singhal S, Vachani A, Antin-Ozerkis D, Kaiser LR and Albelda SM: Prognostic implications of cell cycle, apoptosis, and angiogenesis biomarkers in non-small cell lung cancer: A review. Clin Cancer Res 11: 3974-3986, 2005.

5. Horton JD, Cohen JC and Hobbs HH: Molecular biology of PCSK9: Its role in LDL metabolism. Trends Biochem Sci 32: 71-77, 2007.

6. Park SW, Moon YA and Horton JD: Post-transcriptional regulation of low density lipoprotein receptor protein by proprotein convertase subtilisin/kexin type 9a in mouse liver. J Biol Chem 279: 50630-50638, 2004.

7. Benjannet S, Rhainds D, Essalmani R, Mayne J, Wickham L, Jin W, Asselin MC, Hamelin J, Varret $M$ and Allard D: NARC-1/PCSK9 and its natu-ral mutants: Zymogen cleavage and effects on the low density lipoprotein (LDL) receptor and LDL cholesterol. J Biol Chem 279: 48865-48875, 2004.

8. Peterson AS, Fong LG and Young SG: PCSK9 function and physiology. J Lipid Res 49: 1595-1599, 2008.

9. Abifadel M, Varret M, Rabès JP, Allard D, Ouguerram K, Devillers M, Cruaud C, Benjannet S, Wickham L, Erlich D, et al: Mutations in PCSK9 cause autosomal dominant hypercholesterolemia. Nat Genet 34: 154-156, 2003.

10. Cohen J, Pertsemlidis A, Kotowski IK, Graham R, Garcia CK and Hobbs HH: Low LDL holesterol in individuals of African descent resulting from frequent nonsense mutations in PCSK9. Nat Genet 37: 161-165, 2005.

11. Cohen JC, Boerwinkle E, Mosley TH Jr and Hobbs HH: Sequence variations in PCSK9, low LDL, and protection against coronary heart disease. N Engl J Med 354: 1264-1272, 2006.

12. Seidah NG and Prat A: The biology and therapeutic targeting of the proprotein convertases. Nat Rev Drug Discov 11: 367-383, 2012.

13. Lan H, Pang L, Smith MM, Levitan D, Ding W, Liu L, Shan L, Shah VV, Laverty M, Arreaza G, et al: Proprotein convertase subtilisin/kexin type 9 (PCSK9) affects gene expression pathways beyond cholesterol metabolism in liver cells. J Cell Physiol 224: 273-281, 2010.

14. Ranheim T, Mattingsdal M, Lindvall JM, Holla OL, Berge KE, Kulseth MA and Leren TP: Genome-wide expression analysis of cells expressing gain of function mutant D374Y-PCSK9. J Cell Physiol 217: 459-467, 2008.

15. Zaid A, Roubtsova A, Essalmani R, Marcinkiewicz J, Chamberland A, Hamellin J, Tremblay M, Jacques H, Jin W, Davignon J, et al: Proprotein convertase subtilisin/kexin type 9 (PCSK9): Hepatocyte-specific low-density lipoprotein receptor degradation and critical role in mouse liver regeneration. Hepatology 48: 646-654, 2008.

16. Mbikay M, Sirois F, Mayne J, Wang GS, Chen A, Dewpura T, Prat A, Seidah NG, Chretien M and Scott FW: PCSK9-deficient mice exhibit impaired glucose tolerance and pancreatic islet abnormalities. FEBS Lett 584: 701-706, 2010.

17. Sun XW, Essalmani R, Day R, Khatib AM, Seidah NG and Prat A: Proprotein convertase subtilisin/kexin type 9 deficiency reduces melanoma metastasis in liver. Neoplasia 14: 1122-1131, 2012.

18. Faitova J, Krekac D, Hrstka R and Vojtesek B: Endoplasmic reticulum stress and apoptosis. Cell Mol Biol Lett 11: 488-505, 2006.

19. Chiang LW, Grenier JM, Ettwiller L, Jenkins LP, Ficenec D, Martin J, Jin F, DiStefano PS and Wood A: An orchestrated gene expression component of neuronal programmed cell death revealed by cDNA array analysis. Proc Natl Acad Sci USA 98: 2814-2819, 2001

20. LaCasse EC, Mahoney DJ, Cheung HH, Plenchette S, Baird S and Korneluk RG: IAP-targeted therapies for cancer. Oncogene 27: 6252-6275, 2008.

21. Adams JM and Cory S: The Bcl-2 apoptotic switch in cancer development and therapy. Oncogene 26: 1324-1337, 2007.

22. Pettersson F, Dalgleish AG, Bissonnette RP and Colston KW: Retinoids cause apoptosis in pancreatic cancer cells via activation of RAR-gamma and altered expression of Bcl-2/Bax. Br J Cancer 87: 555-561, 2002.

23. Dufey E, Sepulveda D, Rojas-Rivera D and Hetz C: Cellular mechanisms of endoplasmic reticulum stress signaling in health and disease. 1. An overview. Am J Physiol Cell Physiol 307: C582-C594, 2014. 
24. Yuan T, Luo BL, Wei TH,Zhang L,He BM and Niu RC: Salubrinal protects against cigarette smoke extract-induced HBEpC apoptosis likely via regulating the activity of PERK-eIF $2 \alpha$ signaling pathway. Arch Med Res 43: 522-529, 2012.

25. Stockwell SR, Platt G, Barrie SE, Zoumpoulidou G, Te Poele RH, Aherne GW, Wilson SC, Sheldrake P, McDonald E, Venet M, et al: Mechanism-based screen for G1/S checkpoint activators identifies a selective activator of EIF2AK3/PERK signalling. PLoS One 7: e28568, 2012.

26. Duan Z, Zhao J, Fan X, Tang C, Liang L, Nie X, Liu J, Wu Q and $\mathrm{Xu}$ G: The PERK-eIF2 $\alpha$ signaling pathway is involved in TCDD-induced ER stress in PC12 cells. Neurotoxicology 44: 149-159, 2014.
27. Jiang Q, Li F, Shi K, Wu P, An J, Yang Y and Xu C: Involvement of p38 in signal switching from autophagy to apoptosis via the PERK/eIF2 $\alpha /$ ATF4 axis in selenite-treated NB4 cells. Cell Death Dis 5: e1270, 2014.

28. Liu CY, Schroder M and Kaufman RJ: Ligand-independent dimerization activates the stress response kinases IRE1 and PERK in the lumen of the endoplasmic reticulum. J Biol Chem 275: 24881-24885, 2000

29. Szegezdi E, Logue SE, Gorman AM and Samali A: Mediators of endoplas-mic reticulum stress-induced apoptosis. EMBO Rep 7: 880-885, 2006. 